\title{
BOUNDS FOR THE ELECTRICAL RESISTANCE FOR HOMOGENEOUS CONDUCTING BODY OF ROTATION
}

\author{
István Ecsedi \\ professor emeritus, University of Miskolc, Institute of Applied Mechanics \\ Miskolc-Egyetemváros, 3515 Miskolc, Hungary,e-mail: mechecs@uni-miskolc.hu \\ Ákos József Lengyel
assistant lecturer, University of Miskolc, Institute of Applied Mechanics
Miskolc-Egyetemváros, 3515 Miskolc, Hungary, e-mail: mechlen@ @uni-miskolc.hu \\ Attila Baksa \\ associate professor, University of Miskolc, Institute of Applied Mechanics \\ Miskolc-Egyetemváros, 3515 Miskolc, Hungary, e-mail: mechab@uni-miskolc.hu \\ Dávid Gönczi \\ assistant lecturer, University of Miskolc, Institute of Applied Mechanics \\ Miskolc-Egyetemváros, 3515 Miskolc, Hungary, e-mail: mechgoda@uni-miskolc.hu
}

\begin{abstract}
A mathematical model is developed for the steady-state electric current flow through in a homogeneous isotropic conductor whose shape is a body of rotation. The body of rotation considered is bounded by the coordinate surfaces of an orthogonal curvilinear coordinate system. The equations of the Maxwell's theory of electric current flow in a homogeneous solid conductor body are used to formulate the corresponding electric boundary value problem. The studied steady-state conduction problem is axisymmetric. The determination of the steady motion of charges is based on the concept of the electrical conductance of the conductors the inverse of which is the electrical resistance. The exact (strict) value of the electrical resistance is known only for bodies with very simple shapes, therefore, the principles and the methods that can be used for creating lower and upper bounds to the numerical value of electrical resistance (electrical conductance) are important. The derivation of the upper and lower bound formulae for the electrical conductance of axisymmetric ring-like conductor is based on the two types of Cauchy-Schwarz inequality. The condition of equality of the derived lower and upper bounds is examined. Several examples illustrate the applications of the derived upper and lower bound formulae.
\end{abstract}

Keywords: electrical resistance, steady-state, body of rotation, lower and upper bounds

\section{Introduction}

Electrical resistance of an electrical conductor is a measure of the difficulty to pass a steady electric current through that conductor. In this paper we discuss the concept of electrical resistance and its inverse of electrical conductance. Some bounding formulae will be proven for the electrical conductance. A detailed analysis deals with the case of conductance of body of rotation. The definition of the electrical resistance is based on Ohm's law, it is defined as the ratio of applied voltage to the current. It must be mentioned the paper (Solivérez, 2012), in which a formulation is presented for the equations 
and boundary conditions that describe the steady flow of electric current in a solid conductor under the applied voltage. The linear relationship between the potential difference and electric current across a segment of circuit of arbitrary shape is discussed. The resistance of a sphere is computed as an example.

It must be noticed, that the applied mathematical formalism follows in some detail the principles used in the study (Ecsedi et al., 2020), which were used to derive lower and upper bounds for thermal conduction resistance of a ring-like body. A significant difference in Sections 2 and 3 of this study is the evidence for the lower and upper bound formulae for the general three-dimensional case. The study (Ecsedi et al., 2020) does not describe the mathematical formalism underlying the estimation formulae for general three-dimensional case that can be applied to arbitrary hollow bodies. In this paper the derivations of bounding formulae for body of rotation are based on the general results of Section 2 of the present paper which are different as given in paper (Ecsedi et al., 2020).

Let us consider the steady motion of charges in the conductors shown in Fig. 1. The conductor body occupies the space domain $V$ and its boundary surfaces are separated into four different parts as $\partial V=\partial V_{1} \cup \partial V_{2} \cup \partial V_{3} \cup \partial V_{4}$. The electric potential $U$ on the boundary surface segments $\partial V_{1}$ and $\partial V_{2}$ are prescribed and the boundary surface segments $\partial V_{3}$ and $\partial V_{4}$ are insulated. So that, we have the following boundary conditions (Ecsedi et al., 2020; Solymar, 1984; Landau and Lifshitz, 1963)

$$
\begin{gathered}
U(x, y, z)=U_{i}=\text { constant }, \quad(x, y, z) \in \partial V_{i}, \quad(i=1,2), \\
\mathbf{n} \cdot \nabla U=\frac{\partial U}{\partial n}=0, \quad(x, y, z) \in \partial V_{i}, \quad(i=3,4) .
\end{gathered}
$$

In Eq. (2) $\mathbf{n}$ denotes the outer normal vector of boundary surface $\partial V$. According to Maxwell's theory (Batygin and Toptygin, 1965; Ecsedi, 1999; Wangsness, 1986; Chirgwin et al., 1971; Solymar, 1984; Landau and Lifshitz, 1963) we have next equations for the steady motion of charges

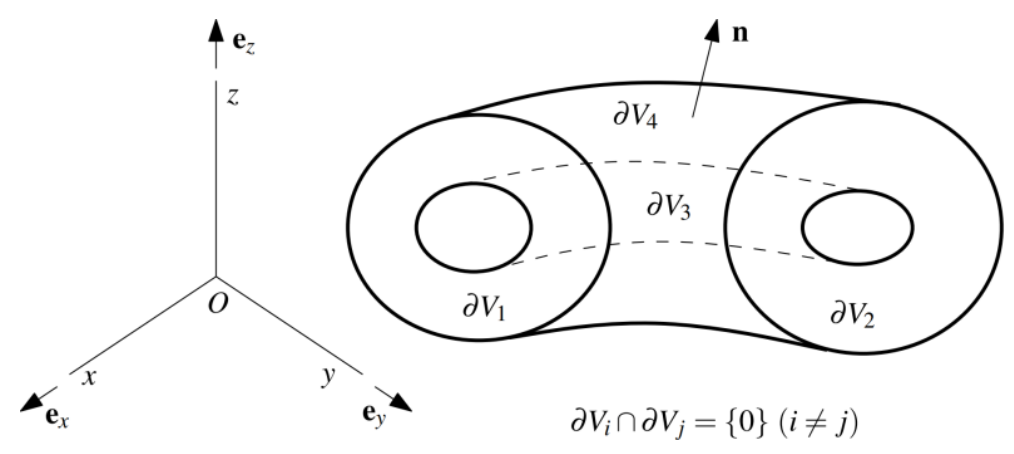

Figure 1. Conductor body bounded by surface segments $\partial V_{i}(i=1,2,3,4)$.

$$
\mathbf{j}=\sigma \mathbf{E}, \quad \nabla \cdot \mathbf{j}=0, \quad \mathbf{E}=-\nabla U .
$$

We note that The International System of Units (SI) is used throughout in this paper. Ohm's law (3) is based on experimental observation which formulates that at constant temperature in isotropic conductor the current density vector $\mathbf{j}$ is proportional to the electric field vector $\mathbf{E}$. From the above equations it follows that

$$
\Delta U=0, \quad(x, y, z) \in V,
$$




$$
\mathbf{j} \cdot \mathbf{n}=-\sigma \mathbf{n} \cdot \nabla U=-\sigma \frac{\partial U}{\partial n}=0, \quad(x, y, z) \in \partial V_{3} \cup \partial V_{4} .
$$

We introduce a new function $u=u(x, y, z)$ by the next definition

$$
U(x, y, z)=\left(U_{2}-U_{1}\right) u(x, y, z)+U_{1}, U_{1} \neq U_{2} .
$$

It is evident that $u=u(x, y, z)$ satisfies the following boundary value problem

$$
\begin{gathered}
\Delta u=0, \quad(x, y, z) \in V, \\
u=0, \quad(x, y, z) \in \partial V_{1}, \quad u=1, \quad(x, y, z) \in \partial V_{2}, \\
\frac{\partial u}{\partial n}=\mathbf{n} \cdot \nabla u=0, \quad(x, y, z) \in \partial V_{3} \cup \partial V_{4} .
\end{gathered}
$$

Here, we note $u=u(x, y, z)$ is unit free. An electric current in the conductor is the continuous passage along that conductor. The constant potential difference between the surfaces $\partial V_{1}$ and $\partial V_{2}$ maintain the steady flow of the electric current. The amount of charge flowing through surface segment $\partial V_{2}$ per unit time is denoted by $I$. The determination of $I$ is based on the next equation (Jackson, 1988; Landau and Lifshitz, 1963)

$$
I=\int_{\partial V_{2}} \mathbf{j} \cdot \mathbf{n} \mathrm{d} A=-\sigma \int_{\partial V_{2}} \mathbf{n} \cdot \nabla U \mathrm{~d} A=\sigma\left(U_{1}-U_{2}\right) \int_{\partial V_{2}} \mathbf{n} \cdot \nabla u \mathrm{~d} A=\sigma\left(U_{1}-U_{2}\right) \int_{\partial V_{2}} \frac{\partial u}{\partial n} \mathrm{~d} A .
$$

We note that the area element of the boundary surface $\partial V$ is denoted by $\mathrm{d} A$. The electrical resistance $R$ of the conductor is defined as (Chirgwin et al., 1971; Solymar, 1984; Landau and Lifshitz, 1963)

$$
R=\frac{U_{1}-U_{2}}{I}=\frac{1}{\sigma \int_{\partial V_{2}} \frac{\partial u}{\partial n} \mathrm{~d} A} .
$$

The conductance $K$ of the conductor body is the inverse of $R$ that is

$$
K=\frac{I}{U_{1}-U_{2}}=\sigma \int_{\partial V_{2}} \frac{\partial u}{\partial n} \mathrm{~d} A
$$

From Eqs. (6-8) it follows that

$$
\int_{V} \Delta u \mathrm{~d} V=\int_{V} \nabla \cdot \nabla u \mathrm{~d} V=\int_{\partial V} \mathbf{n} \cdot \nabla u \mathrm{~d} A=\int_{\partial V_{1}} \mathbf{n} \cdot \nabla u \mathrm{~d} A+\int_{\partial V_{2}} \mathbf{n} \cdot \nabla u \mathrm{~d} A=0,
$$

that is

$$
K=-\sigma \int_{\partial V_{1}} \mathbf{n} \cdot \nabla u \mathrm{~d} A=-\sigma \int_{\partial V_{1}} \frac{\partial u}{\partial n} \mathrm{~d} A
$$


Our aim is to obtain a new form of $K$. For this purpose we start the next identity

$$
\nabla \cdot(u \nabla u)=|\nabla u|^{2}+u \Delta u=|\nabla u|^{2} .
$$

Integrated the above equation over the space domain $V$ and using the Gauss theorem give the next result

$$
\int_{V}|\nabla u|^{2} \mathrm{~d} V=\int_{\partial V} \mathbf{n} \cdot \nabla u \mathrm{~d} A=\int_{\partial V_{2}} \frac{\partial u}{\partial n} \mathrm{~d} A
$$

that is we have

$$
K=\sigma \iint_{V}|\nabla u|^{2} \mathrm{~d} V
$$

\section{Upper bound for $K$, lower bound for $R$}

Theorem 1. If any function $F=F(x, y, z)$ which is continuously differentiable in the space domain $V \cup \partial V$ satisfies the boundary conditions

$$
F(x, y, z)=0, \quad(x, y, z) \in \partial V_{1}, \quad F(x, y, z)=1, \quad(x, y, z) \in \partial V_{2},
$$

then the inequality relation

$$
K \leq \sigma \int_{V}|\nabla F|^{2} \mathrm{~d} V
$$

is true.

Proof. The proof of upper bound formula for $K$ is based on the next Cauchy-Schwarz inequality relation

$$
\left(\int_{V} \nabla F \cdot \nabla u \mathrm{~d} V\right)^{2} \leq \int_{V}|\nabla F|^{2} \mathrm{~d} V \int_{V}|\nabla u|^{2} \mathrm{~d} V .
$$

A simple computation gives

$$
\int_{V} \nabla F \cdot \nabla u \mathrm{~d} V=\int_{\partial V} F \mathbf{n} \cdot \nabla u \mathrm{~d} A-\int_{V} F \Delta u \mathrm{~d} V=\int_{\partial V_{2}} \mathbf{n} \cdot \nabla u \mathrm{~d} A=\int_{V}|\nabla u|^{2} \mathrm{~d} V .
$$

The combination of the inequality relation (20) with Eq. (21) and using formula (17) give the lower bound formula (19). A brief investigation based on Cauchy-Schwarz inequality (20) shows that the sign of equality in relation (19) is valid only if $F(x, y, z) \equiv u(x, y, z)$.

\section{Lower bound for $K$, upper bound for $\boldsymbol{R}$}

Theorem 2. Let $\mathbf{q}=\mathbf{q}(x, y, z)$ be a vector field defined in the space domain $V \cup \partial V$. The identically non-zero vector field $\mathbf{q}=\mathbf{q}(x, y, z)$ satisfies the following equations 


$$
\begin{gathered}
\nabla \cdot \mathbf{q}=0, \quad(x, y, z) \in V, \\
\mathbf{n} \cdot \mathbf{q}=0, \quad(x, y, z) \in \partial V_{3} \cup \partial V_{4},
\end{gathered}
$$

in this case we have

$$
K \geq \sigma \frac{\left(\int_{\partial V_{2}} \mathbf{n} \cdot \mathbf{q} \mathrm{d} A\right)^{2}}{\int_{V} \mathbf{q}^{2} \mathrm{~d} V}, \int_{V} \mathbf{q}^{2} \mathrm{~d} V \neq 0 .
$$

In lower bound formula (24) equality is reached only if

$$
\mathbf{q} \equiv \lambda \nabla u,
$$

where $\lambda$ is an arbitrary constant differently from zero.

Proof. The proof of lower bound formula is based on the next Cauchy-Schwarz inequality relation

$$
\left(\int_{V} \mathbf{p} \cdot \mathbf{q} d V\right)^{2} \leq \int_{V} \mathbf{p}^{2} \mathrm{~d} V \int_{V} \mathbf{q}^{2} \mathrm{~d} V .
$$

Let

$$
\mathbf{p}=\nabla u
$$

be in inequality relation (26). A simple calculation yields the result

$$
\int_{V} \nabla u \cdot \mathbf{q} \mathrm{d} V=\int_{\partial V} u \mathbf{n} \cdot \mathbf{q} \mathrm{d} A-\int_{V} u \nabla \cdot \mathbf{q} \mathrm{d} V=\int_{\partial V_{2}} \mathbf{n} \cdot \mathbf{q} \mathrm{d} A .
$$

Substitution Eq. (28) into Cauchy-Schwarz inequality (26) gives

$$
\left(\int_{\partial V_{2}} \mathbf{n} \cdot \mathbf{q} \mathrm{d} A\right)^{2} \leq \int_{V}|\nabla u|^{2} \mathrm{~d} V \int_{V} \mathbf{q}^{2} \mathrm{~d} V .
$$

From inequality relation (29) we obtain immediately the proof of lower bound formula (24).

\section{Some mathematical relationships for body of rotation}

The considered body of rotation is bounded by the coordinate surfaces of an orthogonal curvilinear coordinate system. The meridian section of the axisymmetric conductor can be seen in Fig. 2. The whole boundary surface of body of rotation $V$ is $\partial V=\partial V_{1} \cup \partial V_{2} \cup \partial V_{3} \cup \partial V_{4}$. The meridian section of $V$ is a simply connected plane domain $A$. The boundary curve of $A$ is $\partial A$ which is divided into four parts as $\partial A=\partial A_{1} \cup \partial A_{2} \cup \partial A_{3} \cup \partial A_{4}$ (Fig. 2). The boundary surface segment $\partial V_{i}$ is obtained from curve $\partial A_{i}$ by a complete rotation of $\partial A_{i}$ about axis $z(i=1,2,3,4)$. The curves $\partial A_{i}(i=1,2,3,4)$ are the coordinate lines of an orthogonal curvilinear coordinate system $(\alpha, \beta)$ defined in the meridian 
plane $(r, z)$ (Fig. 2). Computations are made in the spatial orthogonal curvilinear coordinate system $(\alpha, \beta, \varphi)$. A point $P$ in the space is located in terms of polar angle $\varphi$ of the meridian plane comprising point $P$ and orthogonal curvilinear coordinates $\alpha, \beta$ interpreted in the meridian plane. In the meridian plane setting out by the polar angle $\varphi$ the position vector $\rho$ of point $P$ is

$$
\boldsymbol{\rho}=r \mathbf{e}_{r}+z \mathbf{e}_{z}, \quad r=r(\alpha, \beta), \quad z=z(\alpha, \beta) .
$$

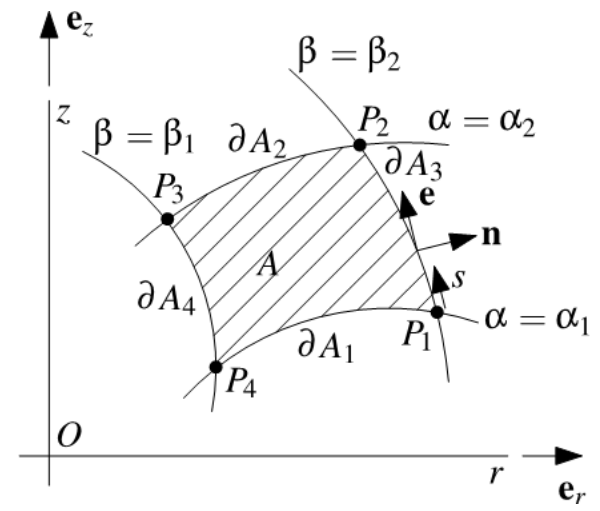

Figure 2. Meridian section of body of rotation

Along lines $\alpha$ and $\beta$ of the orthogonal curvilinear coordinate system $(\alpha, \beta) \alpha=$ constant and $\beta=$ constant respectively (Fig. 3). The tangential unit vector along curve $\beta=$ constant can be written as follows

$$
\mathbf{e}_{\alpha}=\frac{1}{H_{\alpha}} \frac{\partial \mathbf{p}}{\partial \alpha}=\frac{1}{H_{\alpha}}\left(\frac{\partial r}{\partial \alpha} \mathbf{e}_{r}+\frac{\partial z}{\partial \alpha} \mathbf{e}_{z}\right)
$$

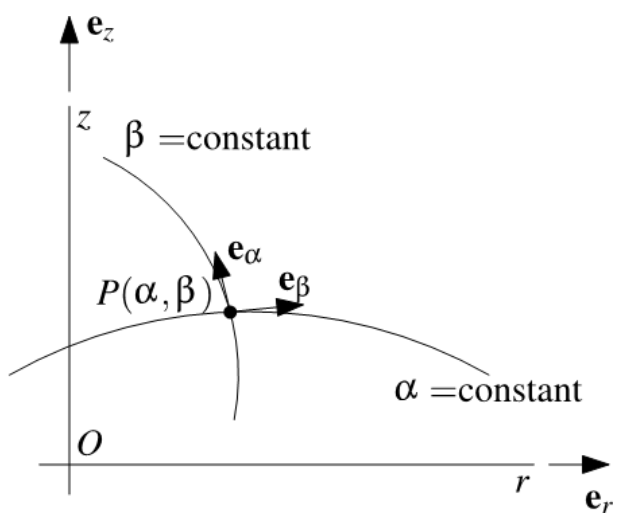

Figure 3. Coordinate lines $\alpha$ and $\beta$

and the tangential unit vector along curve $\alpha=$ constant is

$$
\mathbf{e}_{\beta}=\frac{1}{H_{\beta}} \frac{\partial \boldsymbol{\rho}}{\partial \beta}=\frac{1}{H_{\beta}}\left(\frac{\partial r}{\partial \beta} \mathbf{e}_{r}+\frac{\partial z}{\partial \beta} \mathbf{e}_{z}\right) .
$$


In the formulae above

$$
\begin{aligned}
& H_{\alpha}=\sqrt{\left(\frac{\partial r}{\partial \alpha}\right)^{2}+\left(\frac{\partial z}{\partial \alpha}\right)^{2}} \\
& H_{\beta}=\sqrt{\left(\frac{\partial r}{\partial \beta}\right)^{2}+\left(\frac{\partial z}{\partial \beta}\right)^{2}}
\end{aligned}
$$

are the Lamé's coefficients. The third Lamé coefficient is

$$
H_{\varphi}=r(\alpha, \beta) .
$$

The area element in the plane $r z$ is as follows

$$
\mathrm{d} A=H_{\alpha} H_{\beta} \mathrm{d} \alpha \mathrm{d} \beta,
$$

and volume element of body of rotation is

$$
\mathrm{d} V=2 \pi H_{\alpha} H_{\beta} H_{\varphi} \mathrm{d} \alpha \mathrm{d} \beta .
$$

The orthogonality condition of coordinate lines $\alpha=$ constant and $\beta=$ constant is formulated as

$$
\mathbf{e}_{\alpha} \cdot \mathbf{e}_{\beta}=0,
$$

that is,

$$
\frac{\partial r}{\partial \alpha} \frac{\partial r}{\partial \beta}+\frac{\partial z}{\partial \alpha} \frac{\partial z}{\partial \beta}=0
$$

\section{Electrical conductance of body of rotation}

At first we formulate the electrical conductance of body of rotation in the curvilinear coordinate system $(\alpha, \beta, \varphi)$. To do this, it is needed to compute $|\nabla u|^{2}$ in the orthogonal curvilinear coordinate system $(\alpha, \beta, \varphi)$. Since the considered steady-state problem is axisymmetric we have $u=u(\alpha, \beta)$ and

$$
|\nabla u|^{2}=\frac{1}{H_{\alpha}^{2}}\left(\frac{\partial u}{\partial \alpha}\right)^{2}+\frac{1}{H_{\beta}^{2}}\left(\frac{\partial u}{\partial \beta}\right)^{2} .
$$

Substitution of Eq. (40) into the formula (16) we obtain

$$
K=2 \pi \sigma \int_{\alpha_{1}}^{\alpha_{2} \beta_{2}} \int_{\beta_{1}}\left[\frac{H_{\beta}}{H_{\alpha}}\left(\frac{\partial u}{\partial \alpha}\right)^{2}+\frac{H_{\alpha}}{H_{\beta}}\left(\frac{\partial u}{\partial \beta}\right)^{2}\right] H_{\varphi} \mathrm{d} \alpha \mathrm{d} \beta .
$$

It is evident in the curvilinear coordinates $\alpha, \beta$ and $\varphi F$ depends only on $\alpha$ and $\beta$, from this fact it follows that 


$$
K \leq 2 \pi \sigma \int_{\alpha_{1}}^{\alpha_{2} \beta_{1}} \int_{\beta_{1}}\left[\frac{H_{\beta} H_{\varphi}}{H_{\alpha}}\left(\frac{\partial F}{\partial \alpha}\right)^{2}+\frac{H_{\alpha} H_{\varphi}}{H_{\beta}}\left(\frac{\partial F}{\partial \beta}\right)^{2}\right] \mathrm{d} \alpha \mathrm{d} \beta,
$$

where $F=F(\alpha, \beta)$ satisfies the next boundary conditions

$$
F\left(\alpha_{1}, \beta\right)=0, \quad F\left(\alpha_{2}, \beta\right)=1, \quad \beta_{1} \leq \beta \leq \beta_{2} .
$$

In the present case for the axisymmetric conductor we have

$$
\mathbf{q}=q_{r}(r, z) \mathbf{e}_{r}+q_{z}(r, z) \mathbf{e}_{z},
$$

and

$$
\nabla \cdot \mathbf{q}=\frac{1}{r} \frac{\partial}{\partial r}\left(r q_{r}\right)+\frac{\partial q_{z}}{\partial z}=0 .
$$

Eq. (45) is satisfied if

$$
r q_{r}=-\frac{\partial f}{\partial z}, \quad r q_{z}=\frac{\partial f}{\partial r} .
$$

In Eq. (46) $f=f(r, z)$ is an arbitrary but at least twice continuously differentiable function of its independent variables $r$ and $z$. A simple computation shows that (Fig. 4)

$$
\mathbf{q} \cdot \mathbf{n}=-\frac{1}{r} \frac{\partial f}{\partial z} n_{r}+\frac{1}{r} \frac{\partial f}{\partial r} n_{z}=-\frac{1}{r}\left(\frac{\partial f}{\partial z} \frac{\mathrm{d} z}{\mathrm{~d} s}+\frac{\partial f}{\partial r} \frac{\mathrm{dr}}{\mathrm{d} s}\right)=-\frac{1}{r} \frac{\partial f}{\partial s},
$$

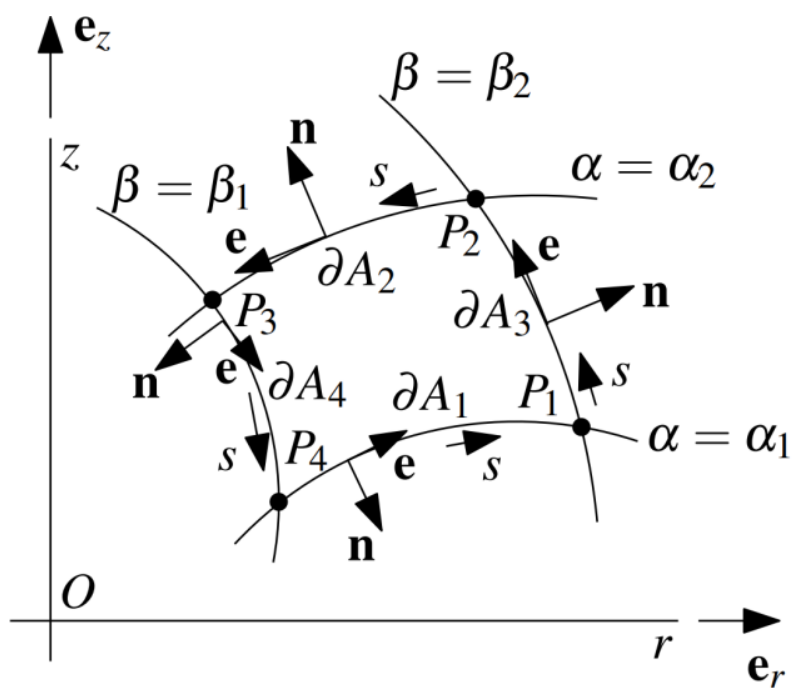

Figure 4. Definition of the unit normal and tangential vectors of boundary curve

where $s$ is an arc-length coordinate defined on the boundary curve $\partial A=\partial A_{1} \cup \partial A_{2} \cup \partial A_{3} \cup \partial A_{4}$. Here, we use the parametric equation of boundary curve $\partial A$. The boundary condition (23) is satisfied on the boundary curve segments $\partial A_{3}$ and $\partial A_{4}$ if 


$$
\begin{aligned}
& f\left(\alpha, \beta_{1}\right)=0, \quad f(r, z)=0 \text { on } \partial A_{3}, \\
& f\left(\alpha, \beta_{2}\right)=1, \quad f(r, z)=1 \text { on } \partial A_{4} .
\end{aligned}
$$

It is evident

$$
\begin{gathered}
\mathbf{q}^{2}=\frac{1}{(r(\alpha, \beta))^{2}}\left[\frac{1}{H_{\alpha}^{2}}\left(\frac{\partial f}{\partial \alpha}\right)^{2}+\frac{1}{H_{\beta}^{2}}\left(\frac{\partial f}{\partial \beta}\right)^{2}\right], \\
\int_{V} \mathbf{q}^{2} \mathrm{~d} V=2 \pi \int_{\beta_{1}}^{\beta_{2} \alpha_{1}}\left[\frac{H_{\beta}}{H_{\alpha} H_{\varphi}}\left(\frac{\partial f}{\partial \alpha}\right)^{2}+\frac{H_{\alpha}}{H_{\beta} H_{\varphi}}\left(\frac{\partial f}{\partial \beta}\right)^{2}\right] \mathrm{d} \alpha \mathrm{d} \beta, \\
\int_{\partial V_{2}} \mathbf{n} \cdot \mathbf{q} \mathrm{d} A=2 \pi \int_{\partial A_{2}} m \cdot \mathbf{q} \mathrm{d} s=2 \pi \int_{\partial A_{2}} \frac{\partial f}{\partial s} \mathrm{~d} s=2 \pi \int_{P_{2} P_{3}} \frac{\partial f}{\partial s} \mathrm{~d} s=2 \pi\left[f\left(P_{3}\right)-f\left(P_{2}\right)\right]=2 \pi .
\end{gathered}
$$

By the use of above obtained equations in the lower bound formula (24) we get the next result

$$
K \geq \frac{2 \pi \sigma}{2 \pi \int_{\beta_{1}}^{\beta_{2}} \int_{\alpha_{1}}\left[\frac{H_{\beta}}{H_{\alpha} H_{\varphi}}\left(\frac{\partial f}{\partial \alpha}\right)^{2}+\frac{H_{\alpha}}{H_{\beta} H_{\varphi}}\left(\frac{\partial f}{\partial \beta}\right)^{2}\right] \mathrm{d} \alpha \mathrm{d} \beta} .
$$

\section{Some upper and lower bound formulae}

From inequality relation (42) by using the function

$$
F=F(\alpha), \quad \alpha_{1} \leq \alpha \leq \alpha_{2}, \quad \beta_{1} \leq \beta \leq \beta_{2},
$$

which satisfies the boundary conditions

$$
F\left(\alpha_{1}\right)=0, \quad F\left(\alpha_{2}\right)=1
$$

the following upper bound may be deduced for $K$

$$
K \leq 2 \pi \sigma \int_{\alpha_{1}}^{\alpha_{2}} N(\alpha)\left(\frac{\mathrm{d} F}{\mathrm{~d} \alpha}\right)^{2} \mathrm{~d} \alpha,
$$

where

$$
N(\alpha)=\int_{\beta_{1}}^{\beta_{2}} H_{\varphi}(\alpha, \beta) \frac{H_{\beta}(\alpha, \beta)}{H_{\alpha}(\alpha, \beta)} \mathrm{d} \beta
$$

By the application of the known results of variational calculus (Weinstock, 1952; Chattopadhyay, 2004) it can be pointed out that the upper bound (56) is the sharpest in the case 


$$
F(\alpha)=\frac{\int_{\alpha_{1}}^{\alpha} \frac{\mathrm{d} \xi}{N(\xi)}}{\int_{\alpha_{1}}^{\alpha_{2}} \frac{\mathrm{d} \xi}{N(\xi)}} .
$$

The substitution of the expression $F=F(\alpha)$ given by Eq. (58) into upper bound formula (56) gives

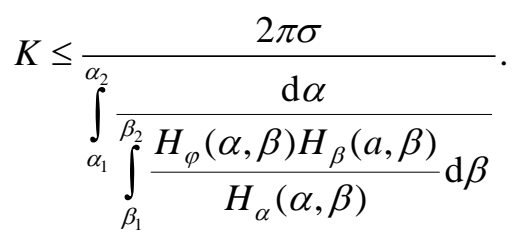

The substitution of the following function

$$
f=f(\beta), \quad \alpha_{1} \leq \alpha \leq \alpha_{2}, \quad \beta_{1} \leq \beta \leq \beta_{2}
$$

which satisfies the boundary conditions

$$
f\left(\beta_{1}\right)=0, \quad f\left(\beta_{2}\right)=1
$$

into the inequality relation (53) provides the next lower bound formula for the electrical conductance

$$
K \geq \frac{2 \pi \sigma}{\int_{\beta_{1}}^{\beta_{2}} n(\beta)\left(\frac{\mathrm{d} f}{\mathrm{~d} \beta}\right)^{2} \mathrm{~d} \beta} .
$$

Here

$$
n(\beta)=\int_{\alpha_{1}}^{\alpha_{2}} \frac{H_{\alpha}(\alpha, \beta)}{H_{\varphi}(\alpha, \beta) H_{\beta}(\alpha, \beta)} \mathrm{d} \alpha .
$$

By using the known results of calculus of variation (Weinstock, 1952; Chattopadhyay, 2004) it can be pointed out that the sharpest lower bound for $K$ from inequality relation (62) is obtained if

$$
f(\beta)=\frac{\int_{\beta_{1}}^{\beta} \frac{\mathrm{d} \xi}{n(\xi)}}{\int_{\beta_{1}}^{\beta_{2}} \frac{\mathrm{d} \xi}{n(\xi)} .}
$$

Inserting of the expression $f=f(\beta)$ given by Eq. (64) into the lower bound formula (62) yields the next result 


$$
K \geq 2 \pi \sigma \int_{\beta_{1}}^{\beta_{2}} \frac{\mathrm{d} \beta}{\alpha_{\alpha_{1}}} \frac{H_{\alpha}(\alpha, \beta)}{H_{\varphi}(\alpha, \beta) H_{\beta}(\alpha, \beta)} \mathrm{d} \alpha
$$

It should be notedthat the correctness of Eqs. (58), (59), (64), (65) follows directly from the theorem presented in the Appendix of this paper, so that the reference to the variational calculus can be omitted.

\section{The condition of equality of the upper bound (59) and lower bound (65)}

Theorem 3. Let

$$
\frac{H_{\varphi}(\alpha, \beta) H_{\beta}(a, \beta)}{H_{\alpha}(\alpha, \beta)}=\frac{\frac{\mathrm{d} Y}{\mathrm{~d} \beta}}{\frac{\mathrm{d} X}{\mathrm{~d} \alpha}}
$$

be. The function $X=X(\alpha)$ depends on only $\alpha$ and the function $Y=Y(\beta)$ depends on only $\beta$. In this case the accurate (strict) value of the electric conductance $K$ is given by the next formula

$$
K=2 \pi \sigma \frac{Y\left(\beta_{2}\right)-Y\left(\beta_{1}\right)}{X\left(\alpha_{2}\right)-X\left(\alpha_{1}\right)} .
$$

Proof. A direct computation shows that if Eq. (66) is valid then the upper and lower bounds (59) and (65) gives the same result which is given by Eq. (67).

The correctness of the following theorem can be easily seen.

Theorem 4. Assuming that

$$
H_{\alpha}(\alpha, \beta)=H_{\beta}(\alpha, \beta), \quad(\alpha, \beta) \in A \cup \partial A
$$

and

$$
r=H_{\varphi}(\alpha, \beta)=h_{1}(\alpha) h_{2}(\beta), \quad(\alpha, \beta) \in A \cup \partial A,
$$

then we have

$$
X(\alpha)=\int \frac{\mathrm{d} \alpha}{h_{1}(\alpha)}, \quad Y(\beta)=\int h_{2}(\beta) \mathrm{d} \beta .
$$

The applications of Theorem 4 are illustrated by examples of Section 9 of this paper.

It must be mentioned that in some cases the exact value of the torsional rigidity of elastic ring-like body is obtained from similar formula as (67) (Ecsedi, 1999). The lower and upper bounds of the torsional rigidity of elastic rings were derived by the application of two minimum principles of elasticity (Ecsedi, 1999). 


\section{Numerical example}

The meridian section of the ring-like body of rotation is shown in Fig. 5. In this example we have

$$
H_{\alpha}=1, \quad H_{\beta}=\alpha, \quad H_{\varphi}=a+\alpha \cos \beta .
$$

Application of the upper bound formula (59) for the present case gives

$$
K \leq \frac{2 \pi \sigma a\left(\beta_{2}-\beta_{1}\right)}{\ln \frac{\alpha_{2}}{\alpha_{1}}-\ln \left|\frac{a\left(\beta_{2}-\beta_{1}\right)+\alpha_{2}\left(\sin \beta_{2}-\sin \beta_{1}\right)}{a\left(\beta_{2}-\beta_{1}\right)+\alpha_{1}\left(\sin \beta_{2}-\sin \beta_{1}\right)}\right|} .
$$

From lower bound formula (65) the following inequality can be derived for the electrical conductance

$$
K \geq 2 \pi \sigma a \int_{\beta_{1}}^{\beta_{2}} \frac{\mathrm{d} \beta}{\ln \frac{\alpha_{2}}{\alpha_{1}}-\ln \left|\frac{a+\alpha_{2} \cos \beta}{a+\alpha_{1} \cos \beta}\right|} .
$$

The application of bounding formulae (72), (73) is illustrated by the next numerical example:

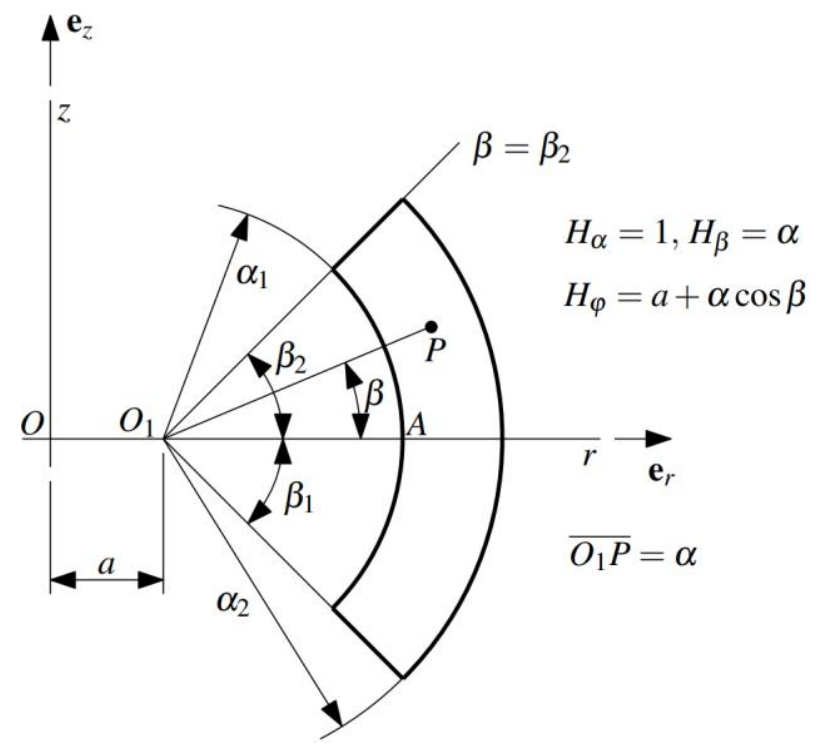

Figure 5. Meridian section of ring-like body of rotation

$$
a=0.9 \mathrm{~m}, \quad \alpha_{1}=0.3 \mathrm{~m}, \quad \alpha_{2}=0.9 \mathrm{~m}, \quad \beta_{1}=-\frac{3 \pi}{4}, \quad \beta_{2}=\frac{3 \pi}{4} .
$$

Substitution of the numerical data (Fig. 5) into the bounding formulae (72), (73) gives the next result

$$
28.3956 \mathrm{~m} \leq \frac{K}{\sigma} \leq 28.6073 \mathrm{~m} .
$$




\section{Determination of electrical conductance}

\subsection{Example}

By the application of Theorem 3 for hollow conductor whose meridian sections are shown in Fig. 6 and Fig. 7 gives the following results for $K$

- for case $a$

$$
X(\alpha)=\alpha, \quad Y(\beta)=\frac{\beta^{2}}{2}, \quad K=\pi \sigma \frac{a_{2}^{2}-a_{1}^{2}}{b_{2}-b_{1}},
$$

- for case $b$

$$
X(\alpha)=\ln \alpha, \quad Y(\beta)=\beta, \quad K=2 \pi \sigma \frac{b_{2}-b_{1}}{\ln \frac{a_{2}}{a_{1}}} .
$$

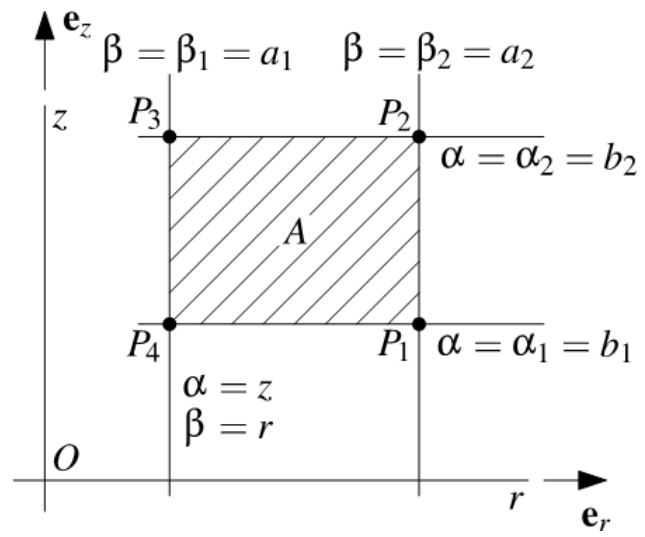

Figure 6. Meridian section of body of rotation bounded by straight lines (case a)

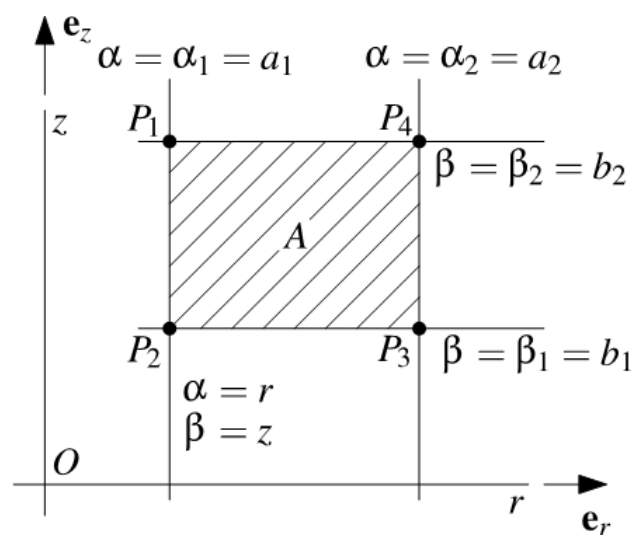

Figure 7. Meridian section of body of rotation bounded by straight lines (case b) 


\subsection{Example}

Figs. 8 and 9 show the meridian sections which are bounded by circles and straight lines - for case $a$

$$
\begin{gathered}
r=\alpha \cos \beta, \quad z=\alpha \sin \beta, \quad H_{\alpha}=1, \quad H_{\beta}=\alpha, \quad H_{\varphi}=\alpha \cos \beta, \\
\overline{O P}_{1}=\overline{O P}_{4}=\rho_{1}, \quad \overline{O P}_{2}=\overline{O P}_{3}=\rho_{2}, \quad X(\alpha)=-\frac{1}{\alpha}, \quad Y(\beta)=\sin \beta,
\end{gathered}
$$

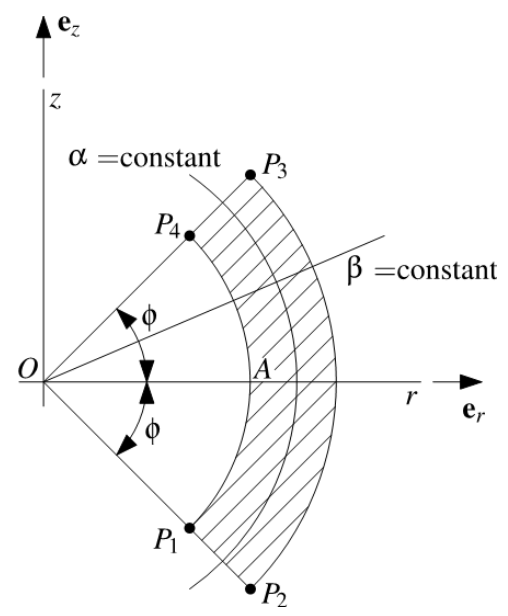

Figure 8. Meridian section of ring-like body bounded by circles and straight lines (case a)

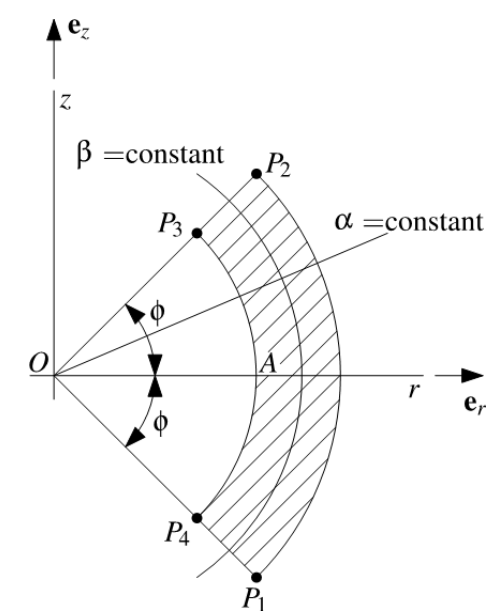

Figure 9. Meridian section of ring-like body bounded by circles and straight lines (case b) - for case $b$

$$
r=\beta \cos \alpha, \quad z=\beta \sin \alpha, \quad H_{\alpha}=\beta, \quad H_{\beta}=1,
$$




$$
\overline{O P}_{3}=\overline{O P}_{4}=\beta_{1}=\rho_{1}, \quad \overline{O P}_{1}=\overline{O P}_{2}=\beta_{2}=\rho_{2}, \quad X(\alpha)=\frac{1}{2} \ln \left|\frac{1+\sin \alpha}{1-\sin \alpha}\right|, \quad Y(\beta)=\beta
$$

By the use of formula (67) the electrical conductance is obtained - for case $a$

$$
K=4 \pi \sigma \frac{\rho_{1} \rho_{2}}{\rho_{2}-\rho_{1}} \sin \phi
$$

- for case $b$

$$
K=4 \pi \sigma \frac{\rho_{2}-\rho_{1}}{\ln \left|\frac{1+\sin \phi}{1-\sin \phi}\right|}, 0<\phi<\frac{\pi}{2} .
$$

Here, it is noted that formula (81) for $\phi=\pi / 2$ gives the electrical conductance of hollow sphere (Batygin and Toptygin, 1965; Jackson, 1988; Solymar, 1984).

\subsection{Example}

Figs. 10 and 11 show the meridian sections whose boundary curves are ellipse and hyperbola arcs.

- For case $a$ (Fig. 10)

$$
r=C \sin \alpha \cosh \beta, \quad z=C \cos \alpha \sinh \beta,
$$

$$
H_{\alpha}=H_{\beta}=C \sqrt{\cos ^{2} \alpha+\sinh ^{2} \beta}, \quad H_{\varphi}=C \sin \alpha \cosh \beta, \quad X(\alpha)=\ln \left|\tan \frac{\alpha}{2}\right|, \quad Y(\beta)=C \sinh \beta .
$$

- For case $b$ (Fig. 11)

$$
\begin{gathered}
r=C \cosh \alpha \sin \beta, \quad z=C \sinh \alpha \cos \beta, \\
H_{\alpha}=H_{\beta}=C \sqrt{\sinh ^{2} \alpha+\cos ^{2} \beta}, \quad H_{\varphi}=C \cosh \alpha \sin \beta, \\
X(\alpha)=2 \arctan (\exp \alpha), \quad Y(\beta)=-C \cos \beta .
\end{gathered}
$$

Application of formula (67) to the cases shown in Figs. 10 and 11 yields the next result - for case $a$ (Fig. 10)

$$
K=2 \pi \sigma C \frac{\sinh \beta_{2}-\sinh \beta_{1}}{\ln \left|\frac{\tan \frac{\alpha_{2}}{2}}{\tan \frac{\alpha_{1}}{2}}\right|},
$$

- for case $b$ (Fig. 11)

$$
K=\pi \sigma C \frac{\cos \beta_{1}-\cos \beta_{2}}{\arctan \left(\exp \alpha_{2}\right)-\arctan \left(\exp \alpha_{1}\right)} .
$$




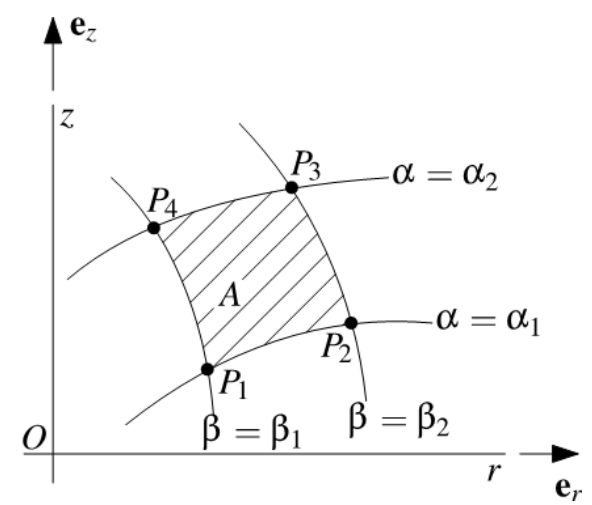

Figure 10. Meridian section of body of rotation bounded by ellipse and hyperbolic arcs (case a)

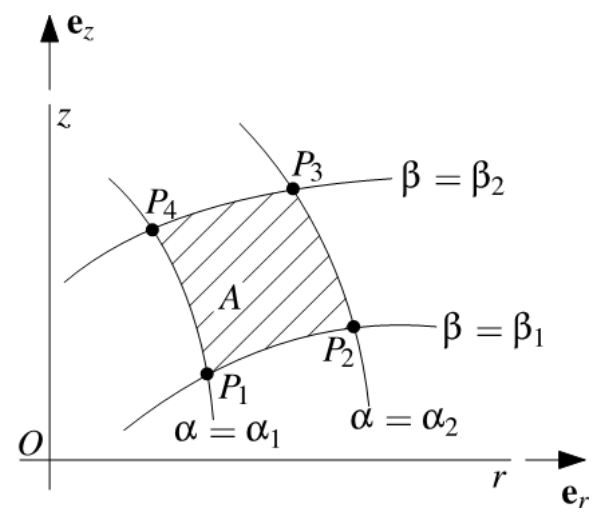

Figure 11. Meridian section of body of rotation bounded by ellipse and hyperbolic arcs (case b)

\subsection{Example}

Fig. 12 shows the meridian section of an axisymmetric ring-like conductor whose meridian section is bounded by parabola arcs. In this example

$$
\begin{gathered}
r=2 C \alpha \beta, \quad z=C\left(\beta^{2}-\alpha^{2}\right), \\
H_{\alpha}=H_{\beta}=2 C \sqrt{\alpha^{2}+\beta^{2}}, \quad H_{\varphi}=2 C \alpha \beta, \\
X(\alpha)=-\frac{1}{\alpha^{2}}, \quad Y(\beta)=C \beta^{2} .
\end{gathered}
$$

The substitution of Eq. (91) into formula (67) gives the next result for electrical conductance

$$
K=2 \pi C \sigma \alpha_{1}^{2} \alpha_{2}^{2} \frac{\beta_{2}^{2}-\beta_{1}^{2}}{\alpha_{2}^{2}-\alpha_{1}^{2}}
$$




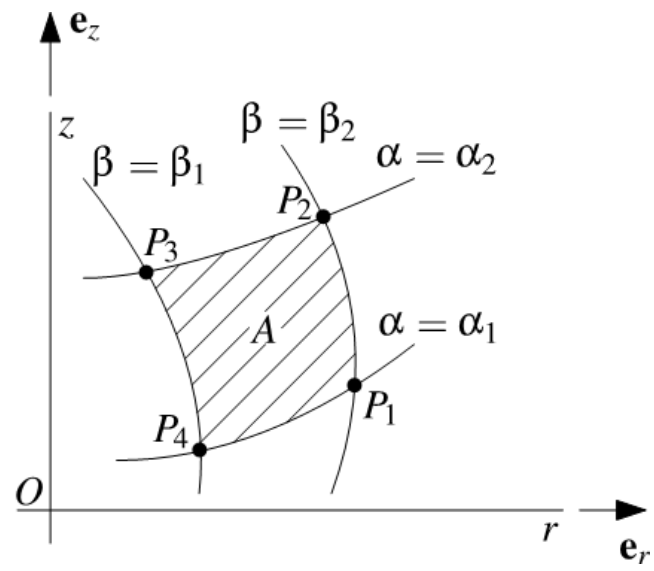

Figure 12. Meridian section of body of rotation bounded by parabola arcs

\section{Conclusions}

Upper and lower bounds for the electrical conductance of homogeneous isotropic axisymmetric ringlike conductor are proven by the application of two types of Cauchy-Schwarz inequality. The discussion of bounding formulae of electrical conductance shows that in some special orthogonal curvilinear coordinate systems the proven upper and lower bounds give the same value for the electrical conductance. In these cases the exact value of electrical conductance can be obtained without solving the boundary value problem of the corresponding field equations of electrostatics. The derived upper and lower bound formulae can be used to check the results of numerical computations obtained by finite element method, boundary element method and by other numerical methods.

\section{Acknowledgements}

This research was supported by the National Research, Development and Innovation Office - NKFIH, K115701. This research was also carried out as part of the EFOP-3.6.1-16-2016-00011 Younger and Renewing University - Innovative Knowledge City - institutional development of the University of Miskolc aiming at intelligent specialisation" project implemented in the framework of the Szechenyi 2020 program. The realization of this project is supported by the European Union, co-financed by the European Social Fund.

\section{Appendix A}

Let us consider the following functional

$$
K[k(x)]=\int_{x_{1}}^{x_{2}} a(x)\left(\frac{\mathrm{d} k}{\mathrm{~d} x}\right)^{2} \mathrm{~d} x,
$$

where $k=k(x)$ is defined for $x_{1} \leq x \leq x_{2}$ and $k=k(x)$ can be continuously differentiable at least twice and

$$
k\left(x_{1}\right)=0, \quad k\left(x_{2}\right)=1 .
$$


The given function $a=a(x)$ satisfies the following conditions

$$
\int_{x_{1}}^{x_{2}}(a(x))^{2} \mathrm{~d} x \neq 0, \quad a(x) \geq 0, \quad x_{1} \leq x \leq x_{2}
$$

In this case we have

\section{Theorem A}

$$
\min _{k(x)} K[k(x)]=K\left[k_{0}(x)\right], \quad k_{0}(x)=\frac{\int_{x_{1}}^{x} \frac{\mathrm{d} t}{a(t)}}{\int_{x_{1}}^{x_{2}} \frac{\mathrm{d} x}{a(x)}}
$$

Proof. Let

$$
k_{1}(x)=k(x)-k_{0}(x), \quad x_{1} \leq x \leq x_{2}
$$

be. It is evident

$$
k_{1}\left(x_{1}\right)=k_{1}\left(x_{2}\right)=0
$$

and

$$
K[k(x)]=K\left[k_{0}(x)\right]+2 \int_{x_{1}}^{x_{2}} a(x) \frac{\mathrm{d} k_{0}}{\mathrm{~d} x} \frac{\mathrm{d} k_{1}}{\mathrm{~d} x} \mathrm{~d} x+K\left[k_{1}(x)\right]
$$

A detailed computation shows that

$$
\int_{x_{1}}^{x_{2}} a(x) \frac{\mathrm{d} k_{0}}{\mathrm{~d} x} \frac{\mathrm{d} k_{1}}{\mathrm{~d} x} \mathrm{~d} x=a\left(x_{2}\right)\left(\frac{\mathrm{d} k_{0}}{\mathrm{~d} x}\right)_{x=x_{2}} k_{1}\left(x_{2}\right)-a\left(x_{1}\right)\left(\frac{\mathrm{d} k_{0}}{\mathrm{~d} x}\right)_{x=x_{1}} k_{1}\left(x_{1}\right)-\int_{x_{1}}^{x_{2}} k_{1}(x) \frac{\mathrm{d}}{\mathrm{d} x}\left[a(x) \frac{\mathrm{d} k_{0}}{\mathrm{~d} x}\right] \mathrm{d} x=0 .
$$

From Eq. (A7) and (A8) it follows that

$$
K[k(x)]=K\left[k_{0}(x)\right]+K\left[k_{1}(x)\right]
$$

that is

$$
K[k(x)] \geq K\left[k_{0}(x)\right]=\frac{1}{\int_{x_{1}}^{x_{2}} \frac{\mathrm{d} x}{a(x)}}
$$




\section{References}

[1] El Dhaba, A. R., Ghaleb, A. F., El-Seadawy, J., Abou-Dina, M. S. (2014). Uncoupled thermomagnetoelastostatics for long cylinders carrying a steady axial electric current by a boundary integral method. A numerical approach. International Journal of Applied Electromagnetics and Mechanics, 46(3), 629-648. https://doi.org/10.3233/JAE-141961

[2] Batygin, V. V., Toptygin, I. N. (1965). Problems in electrodynamics. Academic Press, LondonNew York.

[3] Ecsedi, I., Lengyel, Á. J., Gönczi, D. (2020). Bounds for the thermal conductance of body of rotation. International Review on Modelling and Simulations (IREMOS), 13(3), 185-193. https://doi.org/10.15866/iremos.v13i3.18619

[4] Ecsedi, I. (1999). Bounds for the torsional rigidity of elastic ring. Mechanics Research Communications, 26(4), 445-450. https://doi.org/10.1016/S0093-6413(99)00047-6

[5] Jackson, J. D. (1988). Classical electrodynamics. Wiley and Sons, New York.

[6] Wangsness, R. K. (1986). Electromagnetic fields. Wiley, New York.

[7] Chirgwin, B. H., Plumpton, C., Kilmister, C. W. (1971). Elementary Electromagnetic Theory. Volume 1. Pergamon Press, Oxford. https://doi.org/10.1016/B978-0-08-016080-1.50005-2

[8] Solymar, L. (1984). Lectures on electromagnetic theory: a short course for engineers. Oxford University Press, Oxford.

[9] Landau, L. D., Lifshitz, E. M. (1963). Electrodynamics of Continuous Media. Pergamon Press, Oxford.

[10] Weinstock, R. (1952). Calculus of Variations. McGraw-Hill, New York.

[11] Chattopadhyay, P. K. (2004). Mathematical Physics. New Age International Ltd. Publishers, New Delhi.

[12] Solivérez C. E. (2012). The notion of electrical resistance. www.researchgate.net/publication/234037526. 